\title{
Themenschwerpunkt
}

Z Gerontol Geriat 2020 · 53:644-646 https://doi.org/10.1007/s00391-020-01792-5 Eingegangen: 9. September 2020

Angenommen: 9. September 2020

Online publiziert: 14 . Oktober 2020

(c) Springer Medizin Verlag $\mathrm{GmbH}$, ein Teil von Springer Nature 2020

\author{
Hans-Werner Wahl' · L. Cornelius Bollheimer ${ }^{2}$ \\ ${ }^{1}$ Netzwerk Alternsforschung der Universität Heidelberg und Abteilung für Psychologische \\ Alternsforschung, Psychologisches Institut, Universität Heidelberg, Heidelberg, Deutschland \\ ${ }^{2}$ Medizinische Klinik VI (Altersmedizin), Uniklinik RWTH Aachen, Aachen, Deutschland
}

\section{Gerontologie, Geriatrie und Robotikforschung}

\section{Blick zurück nach vorn}

Robotische Lösungen zu Unterstützung und Anreicherung eines autonomen und zufriedenen Alters, v.a. des sehr hohen Alters, finden im öffentlichen Diskurs der schnell alternden Gesellschaft aktuell sehr viel Aufmerksamkeit. Abbildungen bzw. In-Szene-Setzungen von lächelnden älteren Menschen im direkten Austausch mit den unterschiedlichsten Roboterlösungen sind beinahe täglich in Print-Medien, Wissenschaftssendungen im Hörfunk, auch im TV, in Stellungnahmen von Seniorenverbänden, Alzheimer-Gesellschaften, auf Youtube und zwischenzeitlich auch vielfach in Beiträgen der vorliegenden Zeitschrift sowie Fachbeiträgen und -büchern anzutreffen.

Dabei finden sich - wie Sibylle Meyer et al. in ihrem "guest editorial" schreiben - mehrheitlich realitätsfremde, ScienceFiction-artige Vorstellungen zur Robotik etwa dergestalt, dass ein Stahlkoloss mit allen Aktoren rasselnd eine ältere Dame die Treppe herunterträgt und dabei gequält lächelt. Abgesehen davon, dass flexibles und stabiles Treppengehen immer noch unglaubliche Rechenleistungen voraussetzt und insofern weit entfernt von einer überzeugenden robotischen Lösung ist, kann das jetzt vorliegende Schwerpunktheft der Zeitschrift für Gerontologie und Geriatrie (ZGG) zur assistiven Robotik für ältere Menschen auch dazu beitragen, zumindest in Professionskreisen solche „mentalen Kodierungen" geradezurücken. Vielleicht wird das autonome Fahren als eine Art Hybrid von tradiertem Automobil und Mobilitätsroboter zu einem Erfolgsmodell gerade für ältere Menschen, obwohl in der
Autoindustrie damit eher nicht geworben wird.

Im Beispiel des autonomen Fahrens als unspektakulärer Prototyp assistiver Robotik spiegeln sich Variabilität und Heterogenität robotischer Lösungen auch im Altersbereich wider; diese decken die Funktionen eines stillen Helfers, Interaktionspartners, „companions“ oder Gesundheitsberaters ab und umfassen sowohl autonom vor Ort entscheidende Systeme als auch Lösungen unter ,remote control" im Hintergrund [9-11]. Vor diesem Hintergrund greift es zu kurz, assistive Robotik ausschließlich im Pflegekontext oder in der Geriatrie anzusiedeln, obwohl dies sicherlich einer der wichtigsten Anwendungsbereiche sein wird [6-8]. Mittel- und langfristig geht es beim Einsatz assistiver Robotik ebenfalls um nichtpflegebedürftige alte Menschen im Dienste einer bestmöglichen Autonomie und Lebensqualität sowie um die Nutzung von Präventionspotenzialen.

Dem übergeordneten Thema der (noch mangelnden) Digitalisierung des Alters kommt durch den jetzt veröffentlichten Achten Altersbericht [4] viel Aufmerksamkeit zu. Der Begriff Robotik wird in diesem 100-seitigen Bericht mehr als 100-mal verwendet, was die Bedeutung gerade dieser Thematik im digitalen Kontext unterstreicht. Bemerkenswert ist das im Altersbericht enthaltene eigenständige Kapitel zu Forschungsfragen und -methoden für die aktuellen und zukünftigen digitalen Trends mit Bedeutung für ältere Menschen. Als Kommissionsmitglied (H.-W. W.) darf hierzu bei aller Vertraulichkeit vermerkt werden, dass die Kommission lange darüber dis- 
kutiert hat, ob es ein solches, vielleicht am Ende als „abgehoben“ wahrgenommenes Kapitel überhaupt geben sollte. Vor dem Hintergrund der positiven Rezeption der Bundesregierung - die entsprechende Stellungnahme ist dem eigentlichen Bericht vorangestellt - kann sich in diesem Punkt nicht nur die Kommission bestätigt fühlen. Für die gerontologische und geriatrische Fachgemeinschaft ergeben sich daraus neue Forschungsaufgaben.

Noch erscheint aber assistive Robotik als gerontologisch-geriatrisches Forschungsfeld eher als „spröde“ wahrgenommen $\mathrm{zu}$ werden. Vorherrschend ist eine reservierte Haltung, inwieweit es sich hier überhaupt um einen ernstzunehmenden Forschungstopos handelt. Die Belastbarkeit der Forschungsbefunde und ihre wissenschaftliche Halbwertszeit werden hinterfragt, da sich digitale Technikgenerationen überaus schnell verändern und erst wenige nachhaltige Standards und Richtlinien etabliert sind. Statt anwendungsbezogener Forschung erscheinen kommerziell arbeitende Evaluationsinstitute, vielleicht der Stiftung Warentest oder anderer zertifizierten Prüfstellen, als sinnvollere Alternative.

Solcher Skepsis und forscherischer Passivität stehen die in dieser Ausgabe vorliegenden Beiträge entgegen. Gemeinsam, rasch und flexibel müssen sich die forschende Gerontologie und Geriatrie den Fragen, Möglichkeiten, und dabei sowohl ermutigenden Ergebnissen als auch fehlenden Daten und ablehnender Skepsis in der Öffentlichkeit, in Professionen, und manchmal auch in der Politik, stellen. Wünschenswert wäre insofern:

- eine intensive gerontologische und geriatrische Aufmerksamkeit für das, was sich in der assistiven Robotik forschungsbezogen tatsächlich tut;

- eine ernsthafte und andauernde kritisch wissenschaftliche Auseinandersetzung mit den vorliegenden Studien und Befunden;

- ein komplementäres Einbringen spezifischer Standards und von Fachwissen in die stark technisch ausgerichtete Robotikforschung als methodisches Weiterentwicklungspotenzial der eigenen Fachdisziplin.

Z Gerontol Geriat 2020 ·53:644-646 https://doi.org/10.1007/s00391-020-01792-5

(c) Springer Medizin Verlag GmbH, ein Teil von Springer Nature 2020

\section{H.-W. Wahl • L. C. Bollheimer}

\section{Gerontologie, Geriatrie und Robotikforschung. Blick zurück nach vorn}

\section{Zusammenfassung}

Assistive Robotik als gerontologischgeriatrisches Forschungsfeld scheint bislang eher als "spröde" wahrgenommen zu werden. Vorherrschend ist eine reservierte Haltung, inwieweit es sich überhaupt um einen ernstzunehmenden Forschungstopos handelt. Die Belastbarkeit der Forschungsbefunde und ihre wissenschaftliche Halbwertszeit werden hinterfragt, da sich digitale Technikgenerationen überaus schnell verändern sowie erst wenige nachhaltige Standards und Richtlinien etabliert sind. Hierbei droht die Gefahr, dass in der Gerontologie und Geriatrie Wesentliches verpasst wird, denn das Momentum ist genau jetzt aktiv und jetzt gestaltbar. In der wissenschaftlichen Gerontologie und Geriatrie dürfen diese Entwicklungen nicht verschlafen, sondern müssen mit Pioniergeist aufgegriffen und proaktiv mitgestaltet werden. Dies kann durch weitere Forschungsanstrengungen, Wirken in die Öffentlichkeit hinein sowie die Verwertung von klinischem und pflegerischem Praxiswissen mit jeweils Rückspeisung in Entwicklung und Forschung geschehen.

Schlüsselwörter

Assistive Technologie · Forschung und Entwicklung · Gesundheitswissen, -einstellung und -praxis · Autonomie - Lebensqualität

\section{Gerontology, geriatric medicine and robot research. Look back to the future}

Abstract

Assistive robotics as a gerontological geriatric field of research so far seem to be perceived more as "recalcitrant". Predominant is a reserved attitude as to whether this should be considered a research topic to be taken seriously. The reliability of research results and the scientific half-life period are questioned as digital technique generations change exceedingly rapidly and also only a few sustainable standards and guidelines are established. In this context there is a danger that essential developments will be missed in gerontology and geriatrics, then the momentum is active right now and can now be shaped. In the scientific gerontology and geriatrics these developments should not be missed out on but must be grasped with pioneering spirit and proactively cocreated. This can be achieved by further research endeavors, public activities and the utilization of knowledge from clinical and nursing practice with each having feedback into development and research.

\section{Keywords}

Assistive technology $\cdot$ Research and development $\cdot$ Health knowledge, attitudes, practice - Autonomy - Quality of life
Akademisch entwickelt sich mehr und mehr die Situation, dass zentrale Konzepte der Geriatrie, wie z. B. das der Frailty, in puncto Prävention, Früherkennung, Intervention ohne künstliche Intelligenz (KI) und Robotik nur schwer fruchtbar diskutierbar sind. In gleicher Weise erscheinen in der Gerontologie Forschungsprojekte zu Einsamkeit, Autonomie und Wohlbefinden im späten Leben ohne direkte Bezugnahme auf technologische Kontexte, soziale Medien und „robot companions“ zunehmend unvollständig [12]. Weiter ist der akademische Diskurs über das immer prävalenter werdende Vierte Alter und seine Herausfor- derungen in Bezug auf „agency“, hochwertige und entwicklungsfördernde Pflege sowie Würde $[1,5,13]$ wohl schon bald ohne den thematischen Einbezug von intelligenten Assistenzsystemen - oft robotischer Natur - kaum mehr zu führen.

Es besteht die Gefahr, dass diesbezüglich in der Gerontologie und Geriatrie etwas verpasst wird, denn das Momentum ist genau jetzt aktiv und jetzt gestaltbar:

- Aktuell existiert zumindest „substanziell beginnende Evidenz“ zu Alter, Technik und Robotik.

- Der jetzt erschienene Achte Altersbericht der Bundesregierung zu „Ältere[n] Menschen und Digitalisierung“ 
- ist ein konkreter sowie für Gerontologen ${ }^{*}$ innen und Geriater*innen geeigneter Forschungskompass.

- Die tragischen Auswirkungen sozialer Isolation durch die „coronavirus disease 2019“ (COVID-19) müssen kein einmaliges Ereignis bleiben, sondern können in ähnlicher Form wiederkehren. Hier muss durch eine weiterentwickelte Digitalisierung Vorkehrung getroffen werden (s. COVID-19 und Heime, [2, 3]).

- Schließlich müssen Gerontologen ${ }^{\star}$ innen und Geriater*innen sich eingestehen, dass sog. Digitalpakte bislang nur wenig Konkretes für die 17 Mio. Älteren bzw. 3,4 Mio. Pflegebedürftigen bzw. für ihre Angehörigen und die involvierten Professionellen in Deutschland geleistet haben. Hier sind die Fachgesellschaften aufgefordert, ihre Stimme zu erheben und aktiv das Thema Digitalisierung und Alter mitzugestalten.

\section{Fazit für die Praxis}

- Es ist wünschenswert, dass Themenschwerpunkte wie der jetzt vorliegende zur "assistiven Robotik für ältere Menschen" nicht nur in ihrer wissenschaftlichen Bedeutung gewürdigt werden, sondern auch einen Weckrufcharakter übernehmen.

- Nochmals: In der wissenschaftlichen Gerontologie und Geriatrie dürfen diese Entwicklungen nicht verschlafen werden, sondern, im Gegenteil, sie müssen mit Pioniergeist jetzt (oder nie) aufgegriffen und proaktiv mitgestaltet werden.

- Dies kann durch weitere Forschungsanstrengungen, Wirken in die Öffentlichkeit und die Verwertung von klinischen und pflegerischem Praxiswissen mit jeweils Rückspeisung in Entwicklung und Forschung geschehen.

\section{Korrespondenzadresse}

\section{Prof. Dr. Hans-Werner Wah}

Netzwerk Alternsforschung der Universität Heidelberg und Abteilung für Psychologische Alternsforschung, Psychologisches Institut, Universität Heidelberg

Bergheimer Str. 20, 69115 Heidelberg,

Deutschland

wahl@nar.uni-heidelberg.de

Förderung. Der klinische Lehrstuhl von Herrn Professor Bollheimer befasst sich mit der Zusammenarbeit von Ingenieurswissenschaften und Altersmedizin und wird hierfür von der Robert Bosch Stiftung (32.5.1140.0009.0) gefördert

\section{Einhaltung ethischer Richtlinien}

Interessenkonflikt. H.-W. Wahl und L.C. Bollheimer geben an, dass kein Interessenkonflikt besteht.

Für diesen Beitrag wurden von den Autoren keine Studien an Menschen oder Tieren durchgeführt. Für die aufgeführten Studien gelten die jeweils dort angegebenen ethischen Richtlinien.

\section{Literatur}

1. Baltes PB, Smith J (2003) New frontiers in the future of aging: From successful aging of the young old to the dilemmas of the fourth age. Gerontology 49:123-135. https://doi.org/10.1159/000067946

2. Benzinger $P$, Kuru S, Keilhauer A, Hoch J, Prestel $P$, Bauer JM, Wahl H-W (eingereicht) Auswirkungen der Pandemie auf Pflegekräfte und Bewohner von Pflegeheimen sowie deren Angehörige.ZGerontol Geriat

3. Claßen C, Oswald F, Wahl H-W, Heusel C, Antfang P, Becker C (2010) Bewertung neuerer Technologien durch Bewohner und Pflegemitarbeiter im institutionellen Kontext: Befunde des Projekts BETAGT.Z Gerontol Geriat 43:210-218. https://doi. org/10.1007/s00391-010-0126-5

4. Deutscher Bundestag (2020) Achter Bericht zur Lage der älteren Generation in der Bundesrepublik Deutschland: Ältere Menschen und Digitalisierung - und Stellungnahme der Bundesregierung Drucksache 19/21650 vom 13.08.2020. Deutscher Bundestag, Berlin (https://www.achteraltersbericht.de/bericht)

5. Higgs P, Gilleard C (2015) Rethinking old age: theorising the fourth age. Palgrave Macmillan, London

6. Hülsken-Giesler M, Remmers H (2017) Autonome Assistenzsysteme in der Pflege: Potenziale und Grenzen aus pflegewissenschaftlicher Sicht (PflegeRobot) (Gutachten für den Deutschen Bundestag - vorgelegt dem Büro für TechnikfolgenAbschätzung beim Deutschen Bundestag (TAB))

7. Hülsken-Giesler M, Remmers H (2020) Autonome Assistenzsysteme in der Pflege. Potenziale und Grenzen aus pflegewissenschaftlicher Sicht. V\&R unipress, Göttingen

8. Kehl C (2018) Robotik und assistive Neurotechnologien in der Pflege - gesellschaftliche Herausforderungen. TAB-Arbeitsbericht Nr. 177. Büro für Technikfolgen-Abschätzung beim Deutschen Bundestag (TAB), Berlin
9. Klein B, Graf B, Schlömer IF, Roßberg H et al (2018) Robotik in der Gesundheitswirtschaft. Einsatzfelder und Potenziale. medhochzwei, Heidelberg

10. Mombaur K, Corradi D, Ho-Hoang K-L, Schubert A (2018) Assistenzroboter für eine Steigerung der Mobilität im Alter. In: Funke J, Wink M (Hrsg) Perspektiven der Mobilität. Heidelberger Jahrbücher Online, Bd. 3. University Publishing, Heidelberg, S 193-223 https://doi.org/10.17885/ heiup.hdjbo.2018.0.23824

11. Pu L, Moyle W, Jones C, Todorvic M (2019) The effectiveness of social robots for older adults: a systematic review and meta-analysis of randomized controlled studies. Gerontologist 59:e37-e51. https://doi.org/10.1093/geront/ gny046

12. Schulz R, Wahl H-W, Matthews JT, de Vito Dabbs A, Beach SR, Czaja SJ (2015) Advancing the aging and technology agenda in gerontology. Gerontologist 55:724-734. https://doi.org/10. 1093/geront/gnu07

13. Wahl H (2020) Aging successfully: possible in principle? Possible for all? Desirable for all? Integr Psychol Behav Sci 54:251-268. https://doi.org/10. 1007/s12124-020-09513-8 